\title{
Idiopathic dissecting cerebral aneurysm of the distal anterior cerebral artery in an infant successfully treated with aneurysmectomy: illustrative case
}

\author{
Suguru Nagamitsu, MD, Natsue Kaneko, MD, Toshikazu Nagatsuna, MD, PhD, Hiroaki Yasuda, MD, PhD, Manabu Urakawa, MD, PhD, \\ Masami Fujii, MD, PhD, and Tetsuo Yamashita, MD, PhD
}

Department of Neurosurgery, Yamaguchi Prefectural Grand Medical Center, Hofu, Yamaguchi, Japan

\begin{abstract}
BACKGROUND Idiopathic dissecting cerebral aneurysms (IDCAs) are male dominant but are extremely rare in children. Many IDCAs in children are located in the posterior cerebral artery and the supraclinoid internal cervical artery. No cases of IDCA of the distal anterior cerebral artery (ACA) have been reported.

OBSERVATIONS A previously healthy 7-month-old boy experienced afebrile seizures and presented at the authors' hospital 1 week after the first seizure. He was not feeling well but had no neurological deficits. The authors diagnosed a ruptured aneurysm of the right distal ACA based on imaging results. He underwent emergency craniotomy to prevent re-rupture of the aneurysm. Using intraoperative indocyanine green videoangiography, the authors confirmed peripheral blood flow and then performed aneurysmectomy. Pathological examination of the aneurysm revealed a thickened intima, fragmentation of the internal elastic lamina, and a hematoma in the aneurysmal wall. The authors ultimately diagnosed IDCA because no cause was indicated, including a history of trauma. The boy recovered after surgery and was subsequently discharged with no complications.
\end{abstract}

LESSONS The authors reported, for the first time, IDCA of the distal ACA in an infant. The trapping technique is often used for giant fusiform aneurysms in infants. Indocyanine green videoangiography is useful for evaluating peripheral blood flow during trapping in this case.

https://thejns.org/doi/abs/10.3171/CASE20142

KEYWORDS pediatric aneurysm; idiopathic dissecting cerebral aneurysm; distal anterior cerebral artery aneurysm

Pediatric cerebral aneurysms are rare, and there are even fewer reports of idiopathic dissecting cerebral aneurysms (IDCAs)..$^{1,2}$ No IDCA of the distal anterior cerebral artery (ACA) in infants has been reported previously. We performed aneurysmectomy for a ruptured aneurysm in an infant and diagnosed the lesion as an IDCA based on pathological examination. We report this case with a review of the literature.

\section{Illustrative Case}

\section{History and Examination}

A previously healthy 7-month-old boy presented at our outpatient pediatric clinic 1 week after exhibiting afebrile convulsions, which were clonic and lasted approximately 1 minute. He underwent observation as an outpatient. During his return visit 5 days later, he exhibited frequent vomiting and was therefore referred for further examination. The boy was born normally by vaginal delivery at 39 weeks. There was no history of trauma or serious infection. He did not exhibit signs of liveliness, despite the absence of neurological deficits. The anterior fontanel was $20 \times$ $25 \mathrm{~mm}$ without swelling. Magnetic resonance imaging (MRI) demonstrated a 15-mm mass in the anterior longitudinal fissure (Fig. 1A and B). Overall, the lesion exhibited isointensity on T1-weighted imaging (T1WI) and T2-weighted imaging (T2WI), although some parts exhibited high intensity on T1WI and low intensity on T2WI. The right frontal lobe, which was in contact with the lesion, was impaired. Computed tomography (CT) demonstrated localized bleeding around the lesion. CT angiography indicated a 15-mm fusiform aneurysm in the right A3 segment (Fig. 1C). We theorized that the aneurysm rupture caused the acute symptomatic seizures. Emergency craniotomy was planned to prevent re-rupture of the aneurysm and allow pathological examination.

\section{Treatment}

We performed aneurysmectomy of the right ACA by the interhemispheric approach with the patient under general anesthesia. The

ABBREVIATIONS ACA = anterior cerebral artery; $\mathrm{CT}=$ computed tomography; $I D C A=$ idiopathic dissecting cerebral aneurysm; $\mathrm{MRI}=$ magnetic resonance imaging; $\mathrm{T} 1 \mathrm{WI}=$ T1-weighted imaging; T2WI = T2-weighted imaging.

INCLUDE WHEN CITING Published March 1, 2021; DOI: 10.3171/CASE20142.

SUBMITTED December 7, 2020. ACCEPTED December 30, 2020.

(c) 2021 The authors, CC BY-NC-ND 4.0 (http://creativecommons.org/licenses/by-nc-nd/4.0/). 

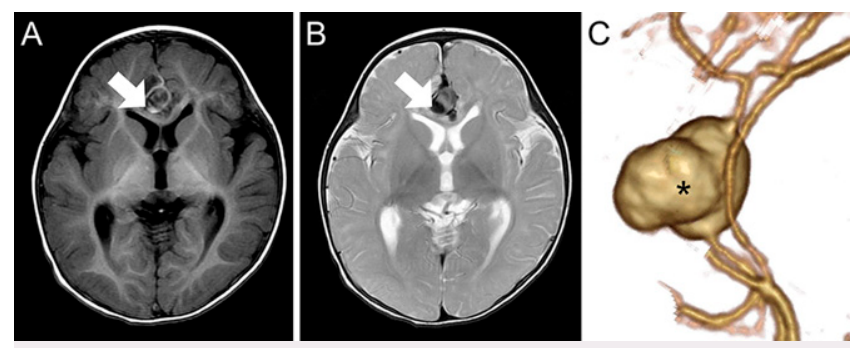

FIG. 1. T1WI (A), T2WI (B), and CT angiography (C) from the patient's first visit. A and B: A $15-\mathrm{mm}$ mass is evident in the anterior longitudinal fissure. Overall, the lesion exhibits isointensity on T1WI and T2WI. Arrows indicate intramural hemorrhage, which exhibits high intensity on T1WI and low intensity on T2WI. C: A 15-mm fusiform aneurysm (asterisk) is visible in the $\mathrm{A} 3$ segment.

dural tension was slightly high. The aneurysm was confirmed by detaching it from between the right frontal lobe and the cerebral falx. A hematoma was located around the aneurysm. The entry and exit of the aneurysm were found by dissecting both ends of the aneurysm. First, the ACA on the proximal side was blocked with a clip (Fig. 2A and B). Indocyanine green videoangiography revealed retrograde blood flow in the distal ACA (Fig. 2C). Next, the ACA on the distal side was blocked with a clip, and the blood flow into the aneurysm was completely occluded. Finally, the aneurysm was resected after we dissected around the aneurysm.

\section{Pathological Characteristics}

The aneurysm wall was composed of thinned adventitia and thickened intima (Fig. 3A). On the whole, the wall was thin. The thickened intima was composed of fibroblasts and collagen fibers. The internal elastic lamina was fragmented, and a slight residue remained (Fig. 3B). Intramural hemorrhage was present, and the intima was similar to an intimal flap in some places (Fig. 3C). A hematoma was also present outside the aneurysm. The aneurysm wall in contact with the hematoma contained signs of neoangiogenesis and inflammatory cells, including neutrophils. No foam cells that would be characteristic of arteriosclerotic changes were present. Furthermore, no bacteria, fungi, or tumor cells were found.

\section{Posttreatment Course}

The patient had no subsequent neurological deficits and regained his vitality after the operation. CT angiography and MRI performed 13 days after surgery confirmed the disappearance of the aneurysm and demonstrated retrograde blood flow in the distal ACA (Fig. 4). The boy had no delayed cerebral ischemia and was discharged 14 days after surgery. He continued to take anticonvulsant agents for acute symptomatic seizures after surgery. We then carefully reduced the use of anticonvulsant agents because he was free from seizures after surgery. He was healthy 6 months after surgery.

\section{Discussion}

\section{Observations}

Pediatric cerebral aneurysms occur rarely compared with aneurysms in adults, accounting for $<4 \%$ of all cerebral aneurysms. ${ }^{1}$ Furthermore, the incidence in infants younger than 1 year is extremely rare. ${ }^{3}$ Factors that contribute to pediatric cerebral aneurysm may include trauma, infection, moyamoya disease, arteriovenous malformation, fibromuscular dysplasia, sickle cell anemia, coarctation
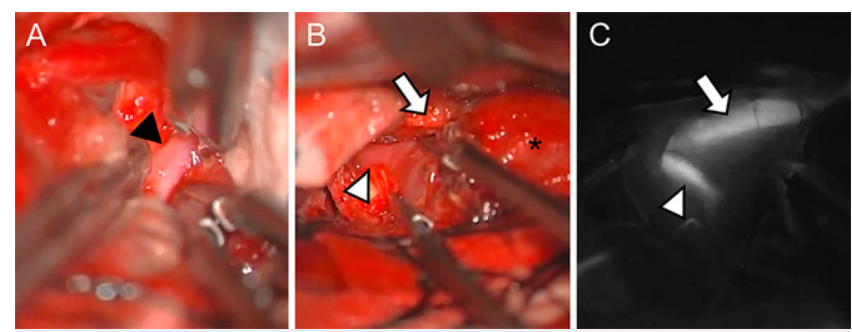

FIG. 2. Intraoperative photographs of the right distal ACA aneurysm. A: The black arrowhead indicates the right ACA on the proximal side, which was blocked with a clip. B: The white arrow indicates the left ACA. The white arrowhead indicates the right ACA on the distal side. The asterisk indicates the aneurysm. C: Indocyanine green videoangiography revealed retrograde blood flow in the right distal ACA on the distal side. The white arrow indicates left ACA and the white arrowhead indicates right ACA on the distal side.

of the aorta, polycystic renal disease, Ehlers-Danlos syndrome, Marfan syndrome, or the like. ${ }^{4}$ It has been reported that dissecting aneurysms after head trauma are the most common pediatric cerebral aneurysms, accounting for $10 \%$ to $39 \%$ of pediatric cerebral aneurysms. ${ }^{5-7}$ Dissecting aneurysms may occur with minor head trauma, ${ }^{8}$ and arterial injury is usually because of acceleration-deceleration between artery and cerebral falx or sphenoid ridge. ${ }^{7,9}$ In our patient, there was no apparent cause of the aneurysm (i.e., trauma, infection, congenital anomaly, etc.). CT angiography revealed that the aneurysm was located in the right $\mathrm{A} 3$ segment, and intraoperative findings showed that the aneurysm was adjacent to the cerebral falx. Therefore, we considered that arterial injury was not due to the acceleration-deceleration between artery and cerebral falx.

Dissecting aneurysms are classified into acute and chronic types, and their pathological findings are distinct. In acute dissection, the internal elastic lamina can be disrupted over a wide area, which leads to intramural hemorrhage. On the other hand, the aneurysm wall can be composed of a thickened intima and a thinned adventitia, and the fragmented internal elastic lamina remains slightly in chronic dissection. Infiltration of inflammatory cells such as macrophages, lymphocytes, and neutrophils is observed on the aneurysm wall. In addition, the collagen fibers in the intima are disrupted in places, causing small dissociations. ${ }^{10}$ We evaluated our patient's aneurysm 1 week after the rupture and encountered pathological findings consistent with chronic dissection. In an experiment that observed the artery with disruption of the internal elastic lamina, it was confirmed that neoplasia of the intima starts to form approximately 1 week after rupture and is complete by approximately 3 months. ${ }^{11}$ This finding suggests that our case did not involve an acute dissection.

Dissecting cerebral aneurysms that have no obvious cause, such as trauma, are called IDCAs. ${ }^{4}$ IDCAs are usually found in the posterior circulation in adults, ${ }^{12,13}$ but pediatric cases have also been reported recently. ${ }^{2}$ Most cases involve boys (68\%), and they show a predilection for the posterior cerebral artery, the supraclinoid internal carotid artery, and the middle cerebral artery. ${ }^{4}$ However, no IDCA of the distal ACA has been reported previously in infants. In our case, the patient was diagnosed as having an IDCA in the distal ACA on the basis of comprehensive evaluation of his medical history, imaging findings, and pathological examination. T1WI can visualize the intramural hemorrhage in the dissecting aneurysm as hyperintensity. In such cases, it has been reported that the aneurysms expand over a short period of 

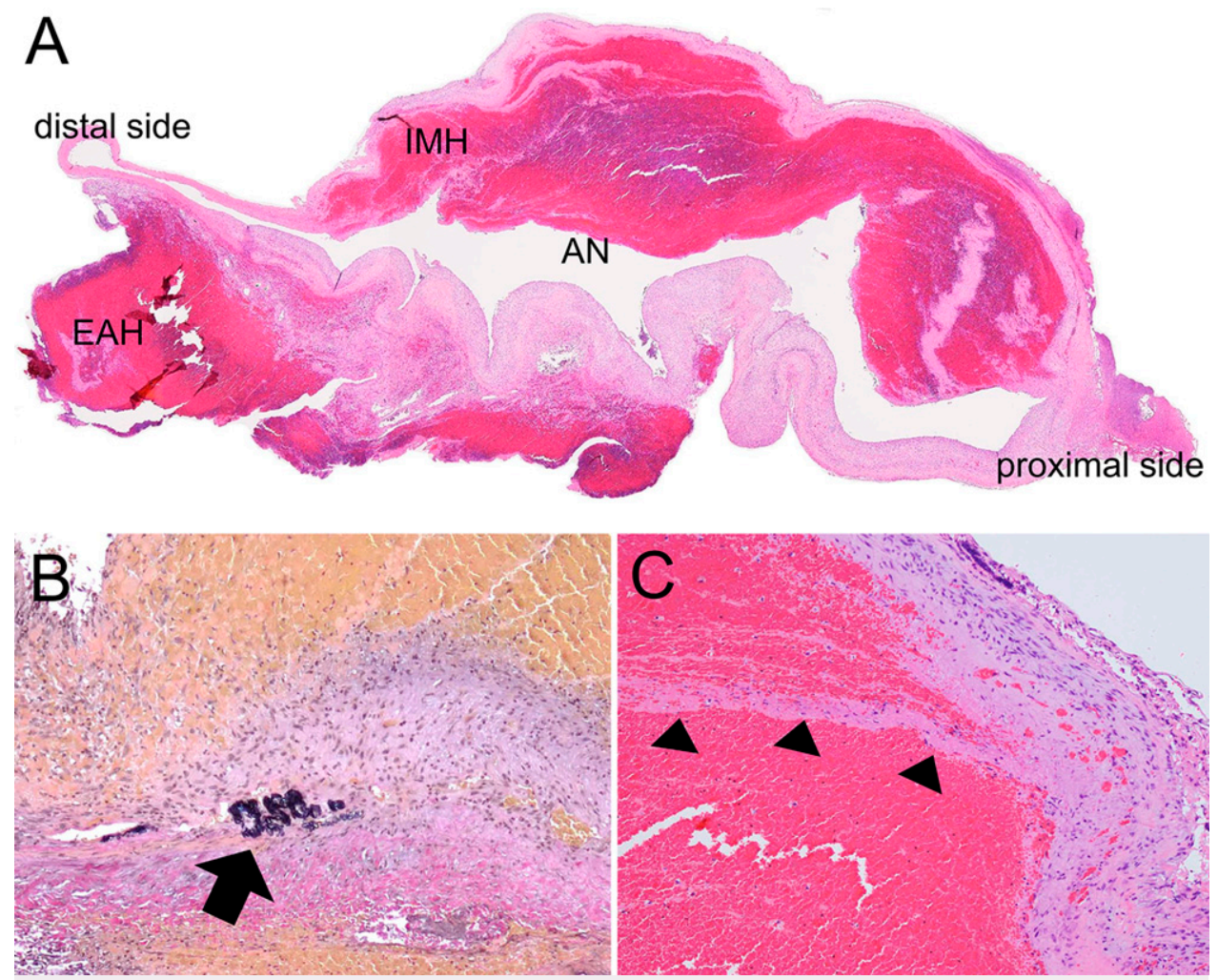

FIG. 3. Pathological images of the aneurysm. A: Original magnification $\times 40$. Hematoxylin and eosin stain demonstrates the coronal section of aneurysm (AN) in a low-power field. The distal ACA is observed on the left, and the proximal ACA is observed on the right. The intramural hemorrhage (IMH) and extraaneurysmal hematoma $(E A H)$ are visible. B: Original magnification $\times 200$. Elastica van Gieson stain demonstrates fragmented internal elastic lamina (arrow). C: Original magnification $\times 200$. Hematoxylin and eosin stain demonstrates the dissected intima-like intimal flap (arrowheads).

time, and most of them have a fatal outcome. ${ }^{14}$ Our case involved a ruptured aneurysm with high intensity on T1WI. Early treatment is considered necessary when a hematoma in the aneurysm wall is visualized by MRI.

Internal trapping is an optional treatment for giant fusiform aneurysms in children. ${ }^{4}$ Trapping is a concern with respect to peripheral ischemic complications. A balloon occlusion test can check ischemia

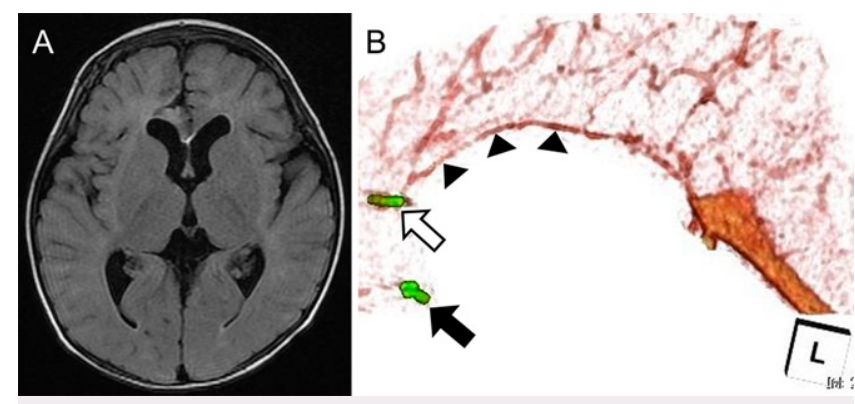

FIG. 4. MRI T2 fluid-attenuated inversion recovery $(\mathbf{A})$ and $C T$ angiography (B) performed 13 days after surgery. A: The aneurysm is properly resected without surrounding damage. B: The black arrow indicates the proximal clip, and the white arrow indicates the distal clip. The arrowheads show retrograde blood flow in the distal ACA. tolerance, but placement of the test occlusion balloon beyond the circle of Willis in infants presents a high risk. ${ }^{7}$ If bypass surgery is attempted in infants, it is difficult to perform vascular anastomosis because of the small diameter of the peripheral artery. Children can tolerate trapping better than adults because of their greater brain plasticity and better compensatory blood supply. ${ }^{15,16}$ Even if symptoms such as paralysis occur after surgery, those symptoms may improve in the long term. ${ }^{7}$ Our case involved a giant fusiform aneurysm of the right ACA in an infant; therefore, we considered trapping to be an optimal technique. Intraoperative indocyanine green videoangiography was performed to assess peripheral blood flow after trapping. We confirmed retrograde blood flow in the peripheral blood vessels in real time with indocyanine green videoangiography, whereas entry into the aneurysm was blocked. Therefore, we predicted that peripheral blood flow would be maintained even if trapped. In fact, no neurological deficits, such as paralysis of the left lower extremity, occurred after the surgery. One problem with this method is that peripheral blood flow cannot be quantified. A specific tool (FLOW 800, Carl Zeiss) can generate blood flow dynamics data by identifying detailed vessel blood flow intraoperatively. ${ }^{17}$ Although this tool has not been used in our hospital, it may be useful for quantifying peripheral blood flow.

\section{Lessons}

We report an IDCA of the distal ACA in an infant. Trapping is a good treatment approach for giant fusiform aneurysms in infants. 
Intraoperative indocyanine green video angiography is useful for evaluating peripheral blood flow during trapping.

\section{References}

1. Amelot A, Saliou G, Benichi S, et al. Long-term outcomes of cerebral aneurysms in children. Pediatrics. 2019;143(6): e20183036.

2. Zhang YS, Wang S, Wang Y, et al. Treatment for spontaneous intracranial dissecting aneurysms in childhood: a retrospective study of 26 cases. Front Neurol. 2016;7:224.

3. Buis DR, Meijer OW, van den Berg R, et al. Clinical outcome after repeated radiosurgery for brain arteriovenous malformations. Radiother Oncol. 2010;95(2):250-256.

4. Levy ML, Levy DM, Manna B. Pediatric Cerebral Aneurysm. StatPearls Publishing; 2020. Accessed August 24, 2020. https:// www.ncbi.nlm.nih.gov/books/NBK537085/

5. Chen R, Zhang S, Guo R, et al. Pediatric intracranial pseudoaneurysms: a report of 15 cases and review of the literature. World Neurosurg. 2018;116:e951-e959.

6. Sorteberg A, Dahlberg D. Intracranial non-traumatic aneurysms in children and adolescents. Curr Pediatr Rev. 2013;9(4): 343-352.

7. Kim B, Lee SK, Terbrugge KG. Endovascular treatment of traumatic intracranial aneurysm in an infant. A case report. Interv Neuroradiol. 2003;9(2):199-204.

8. Munakomi S, Tamrakar K, Chaudhary P, et al. Case report: traumatic anterior cerebral artery aneurysm in a 4-year-old child. F1000 Res. 2015;4:804-812.

9. Buckingham MJ, Crone KR, Ball WS, et al. Traumatic intracranial aneurysms in childhood: two cases and a review of the literature. Neurosurgery. 1988;22(2):398-408.

10. Mizutani T, Kojima H. Clinicopathological study of partiallythrombosed dolichoectatic cerebral aneurysms: a proposed concept of chronic dissecting aneurysm. Article in Japanese. Surg Cereb Stroke (Jpn). 2004;32:331-337.

11. Mitchell GM, McCann JJ, Rogers IW, et al. A morphological study of the long-term repair process in experimentally stretched but unruptured arteries and veins. Br J Plast Surg. 1996;49(1):34-40.
12. Kato M, Tanaka Y, Kuroda T, et al. A case of dissecting aneurysm of the peripheral anterior cerebral artery causing subarachnoid hemorrhage. Article in Japanese. Surg Cereb Stroke (Jpn). 2009;37:288-293.

13. Bradač GB, Peretta P, Stura G, et al. Paediatric dissecting aneurysm of the posterior cerebral artery: case report and review of the literature. Interv Neuroradiol. 2008;14(3):325-330.

14. Nakatomi H, Segawa H, Kurata A, et al. Clinicopathological study of intracranial fusiform and dolichoectatic aneurysms: insight on the mechanism of growth. Stroke. 2000;31(4):896-900.

15. Proust $F$, Toussaint $P$, Garniéri J, et al. Pediatric cerebral aneurysms. J Neurosurg. 2001;94(5):733-739.

16. Ferrante $L$, Fortuna $A, C$ Celli $P$, et al. Intracranial arterial aneurysms in early childhood. Surg Neurol. 1988;29(1):39-56.

17. Ito M, Kuroda S, Nakayama N, et al. Evolution of intraoperative near-infrared indocyanine green video angiography using temporal fluorescence projection methods in cerebrovascular surgery. Article in Japanese. Surg Cereb Stroke (Jpn). 2009;39:40-47.

\section{Disclosures}

The authors report no conflict of interest concerning the materials or methods used in this study or the findings specified in this paper.

\section{Author Contributions}

Conception and design: Nagamitsu. Acquisition of data: Nagamitsu. Analysis and interpretation of data: Nagamitsu. Drafting the article: Nagamitsu, Fujii. Critically revising the article: Kaneko, Nagatsuna, Yasuda, Urakawa, Fujii, Yamashita. Reviewed submitted version of manuscript: all authors. Approved the final version of the manuscript on behalf of all authors: Nagamitsu. Study supervision: Fujii.

\section{Correspondence}

Suguru Nagamitsu: Yamaguchi Prefectural Grand Medical Center, Hofu, Yamaguchi, Japan. neurosurg007.nagamitsu@gmail.com. 